\title{
Estimation of a within-herd transmission rate for African swine fever in Vietnam
}

\author{
To Nga Bui ${ }^{1}$, Nguyen Thi Lan ${ }^{1}$, Jose Canevari ${ }^{2}$, Juan Pablo Villanueva-Cabezas ${ }^{3}$, Pawin \\ Padungtod $^{4}$, Caitlin Pfeiffer ${ }^{2}$, Simon Firestone ${ }^{2}$, Van Phan Le ${ }^{1}$, Madalene Oberin ${ }^{2}$, and \\ Mark Stevenson ${ }^{2}$ \\ ${ }^{1}$ Vietnam National University of Agriculture \\ ${ }^{2}$ University of Melbourne \\ ${ }^{3}$ The Peter Doherty Institute for Infection and Immunity \\ ${ }^{4}$ Food and Agriculture Organization of the United Nations
}

August 14, 2020

\begin{abstract}
We describe results from a panel study in which pigs from a 17-sow African swine fever (ASF) positive herd in Thái Bình province, Vietnam were followed over time to record the date of onset of ASF signs and the date of death from ASF. Our objectives were to: (1) fit a susceptible-exposed-infectious-removed disease model to the data with transmission coefficients estimated using Approximate Bayesian Computation; and (2) provide commentary on how a model of this type might be used to provide decision support for disease control authorities For the outbreak in this herd the median of the average latent period was 10 days (95\% HPD [highest posterior density interval]: 2 to 19 days) and the median of the average duration of infectiousness was 3 days (95\% HPD: 2 to 4 days). The estimated median for the transmission coefficient was 3.3 (95\% HPD: 0.4 to 8.9 ) infectious contacts per ASF-infectious pig per day. The estimated median for the basic reproductive number, $\mathrm{R}_{0}$, was 10 (95\% HPD: 1.1 to 30). Our estimates of the basic reproductive number $R_{0}$ were greater than estimates of $R_{0}$ for ASF reported previously. The results presented in this study may be used to estimate the number of pigs expected to be showing clinical signs at a given number of days following an estimated incursion date. This will allow sample size calculations, with or without adjustment to account for less than perfect sensitivity of clinical examination, to be used to determine the appropriate number of pigs to examine to detect at least one with disease. A second use of the results of this study would be to inform the equation-based within-herd spread components of stochastic agent based and hybrid simulation models of ASF.
\end{abstract}

\section{Hosted file}

VN_ASF_transmission_coefficient_TEXT_v16_SUBMITTED.docx available at https://authorea.com/ users/350754/articles/475680-estimation-of-a-within-herd-transmission-rate-for-african-

swine-fever-in-vietnam 